\title{
Factores pronósticos para artrodesis lumbar
} Prognostic factors for lumbar arthrodesis

\author{
Carlos Alberto Lindado Pacheco ${ }^{a}$ \\ Pontificia Universidad Javeriana, Colombia \\ Santiago GutiérRez ${ }^{\mathrm{b}}$ \\ Pontificia Universidad Javeriana, Colombia \\ Juan Carlos Acevedo González ${ }^{c}$ \\ Pontificia Universidad Javeriana, Colombia
}

a Estudiante de la Especialización en Neurocirugía, Facultad de Medicina, Pontificia Universidad Javeriana, Bogotá, Colombia.

b Médico general, Pontificia Universidad Javeriana. Bogotá, Colombia.

c Director del Departamento de Neurociencias, Facultad de Medicina, Pontificia Universidad Javeriana, Bogotá, Colombia.

Cómo citar: Lindado Pacheco CA, Gutiérrez S, Acevedo González JC. Factores pronósticos para artrodesis lumbar. Univ. Med. 2019;60(1). doi: https://doi.org/10.11144/Javeriana.umed60-1.

\section{RESUMEN}

El dolor de espalda es uno de los síntomas más frecuentes en las instituciones de salud. La neurocirugía desempeña un papel importante en el diagnóstico y tratamiento del dolor de espalda que a menudo resulta en intervenciones quirúrgicas que incluyen la fusión intersomática lumbar entre otras alternativas terapéuticas. Sin embargo, la funcionalidad y los índices de calidad de vida esperados no siempre demuestran una mejora completa, a pesar del tratamiento médico y quirúrgico adecuado. Por lo tanto, los autores realizaron una revisión de la literatura no sistemática para evaluar los elementos más importantes involucrados en las evaluaciones diagnósticas que incluyen: anatomía lumbosacra, procesos patológicos de la columna vertebral que causan dolor de espalda e indicaciones de fusión intersomática lumbar. Finalmente, el papel relevante que tienen los parámetros espinopélvicos en un adecuado planeamiento quirúrgico. Esta revisión de la literatura no sistemática tiene como objetivo establecer la evaluación más contribuyente de los factores espinopélvicos antes de la cirugía para mejorar la selección de pacientes que se someten a tratamiento quirúrgico y, por lo tanto, mejorar su funcionalidad y los índices de calidad de vida.

\section{Palabras clave}

artrodesis; lumbalgia; balance postural.

\section{ABSTRACT}

Back pain is one of the most frequent presenting symptoms in healthcare institutions. Neurosurgery plays a major role on diagnosis and treatment of back pain that often results in surgical interventions that includes lumbar interbody fusion among other therapeutic alternatives. Nevertheless, functionality and expected quality of life indexes do not always demonstrate full improvement despite adequate medical and surgical treatment. Therefore, the authors made a non-systematic literature review in order to assess the most important elements involved on diagnostic assessments that include: lumbosacral anatomy, spine pathological processes that result in back pain, indications for lumbar interbody fusion and finally, the relevant part that spinopelvic balance parameters plays for adequate planning for surgery treatment. This nonsystematic literature review aims to establish the most contributing assessment spinopelvic factors prior surgery in order to improve selection of patients that undergo surgical treatment and therefore, improve their functionality and quality of life indexes. 
Keywords

arthrodesis; low back pain; postural balance.

\section{Introducción}

El dolor lumbar en una patología frecuente y prevalente. Existen cifras que estiman que el $90 \%$ de las personas en algún momento de su vida han experimentado un episodio de dolor lumbar. De este porcentaje, aproximadamente, el $20 \%$ se van a volver permanentes y requerirán múltiples intervenciones para su manejo (1). Dentro de las opciones de manejo, el número de artrodesis lumbares ha aumentado en los países desarrollados. Por ejemplo, en Estados Unidos se pasó de realizar 204.000 cirugías de fusión espinal en 1998 a 457.000 en 2011 (2). Sin embargo, se ha evidenciado que en algunos pacientes el porcentaje de éxito - expresado en términos de dolor controlado y calidad de vida posterior al tratamiento quirúrgico- alcanza tan solo un porcentaje alrededor del 30\%-40\% (3). Por ello es necesario determinar qué factores pueden influir en el éxito de la cirugía de columna.

\section{Métodos}

Se llevó a cabo una revisión no sistemática de la literatura sobre las indicaciones más frecuentes de artrodesis lumbar. Así mismo, se revisaron los conceptos básicos de valoración biomecánica con énfasis en el balance espinopélvico. Posteriormente, se analizó cómo se desempeñan los músculos paravertebrales, la articulación sacroliaca, la sínfisis púbica, la enfermedad facetaría y la importancia del uso de escalas de discapacidad.

\section{Marco teórico}

En el abordaje diagnóstico y terapéutico del paciente con patología espinal son frecuentes los análisis simplificados que solo tienen en cuenta, de forma independiente, las alteraciones anatómicas y funcionales. Por tal motivo, es importante recalcar el papel que tiene el concepto de unidad lumbosacrococcígea, entendido en términos del abordaje global e integral que tienen las diferentes estructuras musculo-óseo-ligamentarias que interactúan desde el punto de vista biomecánico. Dentro de este tipo de análisis existen patrones biomecánicos nuevos que pueden ser determinantes en la evaluación prequirúrgica de los pacientes con artrodesis, para así, en un futuro, implementar una herramienta que permita saber qué pacientes van a tener un mejor pronóstico en el momento de ser llevado a artrodesis lumbar.

El dolor lumbar se debe entender como aquella manifestación desagradable en la región superior a los pliegues glúteos e inferior a la región costal. Un episodio de dolor lumbar agudo puede estar presente en el 75\%-85\% (1) de los individuos y configurar una de las causas más frecuentes de ausencia laboral. Se considera que aquellos individuos quienes han experimentado el dolor durante más de 3 meses cumplen criterios para denominarse dolor lumbar crónico. La importancia del manejo de esta patología radica en su recurrencia y en las manifestaciones que trae consigo para la sociedad (costo de los tratamientos, pérdida de productividad y pagos por indemnización).

En algunos estudios, hasta el 20\% de los pacientes con lumbago que cumplen criterios de cronicidad y que son llevados a cirugía pueden llegar a requerir un nuevo procedimiento. Dentro de las posibles explicaciones para estos resultados está la sacroileitis, por la persistencia del síntoma y puede estar presente hasta en el 30\%-40\% de los casos tratados con artrodesis lumbar (4), o la enfermedad facetaria lumbar, que también puede presentarse frecuentemente después de un tratamiento quirúrgico (18\%-40\%) (2,3). Teniendo en cuenta estos resultados, los pacientes que vayan a ser llevados a artrodesis lumbar requieren una valoración integral en la cual se descarten otras etiologías secundarias que expliquen la cronicidad del dolor y mejoren el pronóstico de funcionalidad y calidad de vida de los pacientes con enfermedad degenerativa vertebral. 


\section{Indicaciones de la artrodesis lumbar}

La cirugía de columna ha pasado por diferentes etapas. Inicialmente, se realizaban procedimientos descompresivos sobre las raíces nerviosas y el canal medular. Posteriormente, con el advenimiento del concepto de inestabilidad vertebral, apareció la artrodesis como una alternativa para evitar el movimiento anormal que se manifiesta en forma de dolor refractario. Dentro de las patologías degenerativas que tienen indicación de artrodesis lumbar se encuentran:

Hernia discal recurrente: estudios poblacionales han informado que pacientes con dolor radicular con pocos síntomas axiales que fueron llevados a cirugía por herniación discal recurrente tienen un 25\% de riesgo adicional de requerir cirugía adicional en los siguientes 10 años. Este riesgo se disminuye si el procedimiento quirúrgico inicial fue con fusión de los segmentos afectados (5).

Canal lumbar estrecho: aquellos pacientes que cursan con estenosis lumbar con evidencia de inestabilidad espinal se benefician de cirugías con artrodesis adicional a los procedimientos descompresivos habituales. Es importante tener en cuenta que aquellos pacientes que son llevados a cirugía descompresiva donde hay afectación de las pars interarticulares o resección facetaría unilateral mayor al $75 \%$ o del $50 \%$ bilateral tienen mayor riesgo de requerir fusión en el futuro.

Espondilolistesis: el desplazamiento vertebral, independiente de su grado y etiología, que es refractario al manejo conservador y se torna sintomático (dolor axial somático crónico o radicular), requiere cirugía con fusión vertebral en varias ocasiones por vía anterior y posterior, en especial cuando las pars interarticulares están afectadas. Algunos estudios de pacientes adultos con espondilólisis sin listesis refractaria al manejo farmacológico durante 6 meses a un año son llevados a artrodesis lumbar (4).

Deformidad: la presencia de patologías deformantes como la escoliosis requieren tener en cuenta la etiología (degenerativa, congénita, neuromuscular, idiopática, iatrogénica), la magnitud y progresión de la curva, los síntomas, el grado de limitación y la respuesta al manejo médico conservador. Pero en un porcentaje importante requieren manejo quirúrgico con artrodesis multinivel.

Enfermedad discal degenerativa: existe evidencia de los últimos años en la cual pacientes con discopatía refractaria a manejo conservador con más de 6 meses de evolución son candidatos a manejo quirúrgico y así mejorar sus índices de calidad de vida. En aquellos pacientes más jóvenes con dolor discogénico corroborado por discografía que son refractarios a manejo médico y percutáneo también son candidatos para cirugía (6).

\section{Evaluación biomecánica}

El concepto de balance espino-pélvico surge de diferentes estudios en los cuales se evidencia la importancia de las interacciones dinámicas simultáneas de la columna vertebral con la pelvis durante la bipedestación y la actividad física. Estos parámetros se han logrado objetivar radiológicamente para garantizar su medición estandarizada y tenerse en cuenta como predictores para la persistencia de dolor en casos donde estén alterados y no se corrijan (7). Algunos de los conceptos más empleados son:

Balance sagital: es la condición de la columna normal caracterizada por mantener una lordosis cervical, cifosis dorsal y lordosis lumbar que permite una postura erecta, con un mínimo de actividad muscular y mínima deformidad de tejidos blandos. Se evalúa al realizar una plomada en el plano sagital, iniciada desde el centro del cuerpo de $\mathrm{C} 7$, pasando sobre el promontorio sacro en un rango de $2 \mathrm{~cm}$ desde su porción más anterior. Se considera positivo cuando esta línea se proyecta anterior al promontorio, y negativo cuando se encuentra posterior a este. En balances positivos, hay una pérdida de lordosis lumbar y aumento de cifosis torácica; ocurre lo contrario con cifras negativas. Este balance se modifica de forma permanente en la columna lumbar: aumenta la lordosis o se rectifica al trabajar sincrónicamente con la base pélvica, 
para distribuir cargas mecánicas y evitar con esto la aparición del dolor (8).

Balance coronal: constituye la relación que tienen los diferentes segmentos de la columna vertebral cuando se valora en el plano coronal. Es frecuente observar en los pacientes con enfermedad discal degenerativa la aparición de escoliosis o listesis laterales que deben ser corregidas en aquellos pacientes que van a ser llevados a cirugía. Sin embargo, tiene menos estudios rigurosos respecto a su importancia en las valoraciones de paciente con dolor lumbar crónico que el balance sagital. La medida que se utiliza es la línea sacra central vertical. Este parámetro se mide trazando una línea a nivel de la apófisis espinosa de C7 y se proyecta vertical para evaluar si coincide con las apófisis espinosas sacras. Se considera un balance coronal positivo cuando hay un desplazamiento de la línea hacia la derecha, y negativo cuando es hacia la izquierda (8).

La incidencia pélvica (PI): se define como el ángulo que se forma entre una línea trazada desde el centro de la cabeza femoral hasta un punto medio en el promontorio sacro y una segunda línea perpendicular a este último punto. Su valor normal varía entre 50 y 55 grados (8). Es un índice importante que suele ser constante. Aunque existen reportes de su aumento durante la infancia en el proceso de aprendizaje de la marcha, es un parámetro constante que no se modifica incluso en pacientes con alteración del balance espino-pélvico. Se ha encontrado que es más importante la relación que mantiene con la lordosis lumbar que un valor absoluto.

La inclinación pélvica (PT): constituye otro parámetro modificable o dinámico, pues cambia a partir del movimiento de la pelvis y la edad, con un gran impacto en la lordosis lumbar. Un aumento de la inclinación pélvica representa un mecanismo compensatorio transitorio con una mayor carga fisiológica que puede manifestarse como dolor lumbar crónico (8). Se mide como el ángulo entre una línea que va desde el centro femoral hasta un punto medio localizado en el platillo terminal del sacro y una segunda línea sobre el trayecto de una línea vertical, que pasa también sobre la cabeza femoral.
Tiene valores normales hasta de 20-30 grados, aunque algunos estudios de pacientes llevados a artrodesis evidencian que ángulos inferiores a 20 grados mejoran la calidad de vida del paciente.

La inclinación sacra (SS): es otro parámetro empleado que se define como el ángulo formado entre una línea que se traza paralela al eje del promontorio sacro y una segunda línea en el horizonte, justo superior al sacro. Hace parte de los parámetros dinámicos en estrecha relación con la incidencia pélvica, ejemplificado en la siguiente ecuación $(\mathrm{PI}=\mathrm{SS}+\mathrm{PT})(8,9)$.

Teniendo en cuenta esta relación estrecha entre la pelvis, la columna vertebral y su funcionamiento dinámico, no es infrecuente encontrar casos en los cuales pacientes que son llevados a procedimientos quirúrgicos sin tener un funcionamiento íntegro de esta unidad espino-pélvica desarrollen disfunción de estructuras adyacentes como la articulación sacroiliaca y manifiesten síndromes dolorosos recurrentes asociados a falla terapéutica y tornen sombrío el pronóstico de algunos pacientes llevados a artrodesis lumbar.

\section{Músculos paravertebrales}

La musculatura en la región lumbar constituye el segundo grupo muscular más grande del cuerpo humano después del cuádriceps femoral, y constituye un papel fundamental en la estabilidad y movilidad espinal (10). Se encuentran organizados en dos categorías: los músculos extrínsecos y los músculos intrínsecos. Dentro de los primeros se encuentra el dorsal ancho y los serratos, que tienen una función más sobre la movilidad apendicular que axial. Los intrínsecos se encargan de regular el tono y la movilidad de la columna. Están inervados por los ramos posteriores de los nervios espinales y se clasifican en tres capas: una superficial (sacroespinalis, formado por el longuísimo y el iliocostal), una media (multífidos) y una profunda (interespinosos, intertransversos y rotadores). Esta musculatura intrínseca representa un área de superficie en cortes axiales de 10 a $25 \mathrm{~cm}^{2}$. 
Músculos multífidos: se originan en las láminas y cuerpos mamilares de las vértebras lumbares. Tienen como función la extensión, la rotación y la flexión lateral.

Músculos intertransversos e interespinosos: conectan las apófisis transversas y espinosas respectivamente de dos cuerpos vertebrales adyacentes. Tienen funciones relacionadas con el posicionamiento espinal a través de la propiocepción.

Músculos erectores espinales: corresponde a la capa más superficial de los paravertebrales. El origen del longuísimo lumbar es en las apófisis transversas lumbares y torácicas inferiores. El ileocostal se origina en el ángulo formado por las costillas inferiores y el cuarto lateral de las apófisis espinosas transversas lumbares. La función de este grupo muscular es principalmente evitar los arcos de movimientos excesivos.

Se han descrito asociaciones en la literatura entre el grado de insuficiencia muscular con la aparición de dolor lumbar crónico (11). Sin embargo, no existen investigaciones claras que expliquen la razón fisiopatológica detrás de este evento. Se han realizado estudios donde el tamaño, la perfusión y el grado de infiltración grasa muscular tiene relación con la aparición de dolor lumbar $(12,13)$, aunque se requieren cohortes prospectivas con mayor número de pacientes para evitar interpretaciones erróneas en razón de posibles variables de confusión.

Algunos estudios imagenológicos de resonancia magnética evidencian que la medición del grado de atrofia muscular (cuantificado por medición longitudinal o del área transversa en un corte axial) tiene limitaciones. Debido principalmente a que el proceso de atrofia muscular viene acompañado de forma simultánea por degeneración grasa. En un estudio realizado en Dinamarca, por Kjaer et al. (10), se encontró infiltración grasa en el $81 \%$ de los adultos en comparación con el $14 \%$ en adolescentes y se obtuvo una relación directamente proporcional con la presencia de dolor lumbar.

\section{La articulación sacroiliaca}

Dentro del abordaje en urgencias del dolor lumbar es frecuente encontrarse con inflamación de la articulación sacroiliaca. Se han descrito entidades en reumatología, como las espondilo-artropatías seronegativas, que debutan principalmente con afectación de esta articulación. Sin embargo, la alteración en el balance permanente que debe existir entre la columna vertebral y la pelvis (para garantizar un funcionamiento adecuado en bipedestación y en la marcha) es una causa importante de disfunción sacroiliaca.

En varios estudios se ha descrito una prevalencia de disfunción de la articulación que oscila entre el $10 \%$ y el $30 \%$ (4). Además, se han descrito muchas maniobras semiológicas dirigidas al diagnóstico de esta patología y la Asociación Internacional para el Estudio del Dolor ha intentado aproximarlas para objetivar el diagnóstico. Sin embargo, varias de estas pruebas todavía tienen valor diagnóstico indeterminado. En otras oportunidades se realiza el diagnóstico de forma retrospectiva en aquellos pacientes que son llevados a manejo percutáneo para tratamiento de síndromes dolorosos persistentes.

Inicialmente, Acevedo y Quintero (14) llevaron a cabo un estudio piloto con 68 pacientes, el cual evidenció que el test de Patrick (sensibilidad del 63\%-100\% y especificidad del $77 \%$ ), el test de Gaenslen (sensibilidad del $71 \%$ y especificidad del $26 \%$ ) y el test de Yeomans tienen el mejor rendimiento diagnóstico de la defunción sacroiliaca. Así mismo, tuvo en cuenta la alta especificidad que tiene la gammagrafía ósea (índices sacroiliacos mayor a 1,5) como predictor imagenológico de la enfermedad. A partir de estos resultados, se hizo una búsqueda de la literatura de los signos más comúnmente empleados para diagnosticar esta patológica. A continuación, un grupo de expertos del Hospital San Ignacio seleccionaron las maniobras diagnósticas más representativas y se evaluaron en 22 pacientes teniendo como estándar de referencia (gold standard) comparativo la respuesta al bloqueo selectivo de la articulación sacroiliaca. Como resultado 
de este estudio, dos características clínicas referidas por el paciente, el test de Laguerre, el test de balanceo sacroiliaco y el test de Yeomans (sensibilidad mayor al 80\%) fueron las maniobras con mejor sensibilidad. Los test con mayor especificidad diagnóstica $(\geq 80 \%)$ fueron el test de Lewitt, el test de Gillet y el test de Piedallu. Ello permitió diseñar la primera escala diagnóstica en la literatura internacional de disfunción de la articulación sacroilica, denominada SI-5 (sensibilidad del $73 \%$ y especificidad del $71 \%$ ).

Se ha encontrado que la enfermedad sacroiliaca es una entidad frecuente en pacientes sometidos a artrodesis lumbar de forma prequirúrgica, además de presentarse en el postoperatorio de pacientes llevados a artrodesis lumbar con fusión del segmento sacroccígeo, en especial cuando requieren fusión de múltiples niveles (15). Por ello es importante su diagnóstico y manejo oportuno para mejorar los desenlaces quirúrgicos.

\section{Sínfisis púbica}

La sínfisis púbica es una estructura fibrocartilaginosa que se encarga de mantener en estrecho contacto los huesos pélvicos, en especial durante la actividad. Esta articulación se encuentra reforzada por estructuras músculoligamentarias que la mantienen en su posición. Dentro de estos ligamentos el arcuato es el que contribuye más para contrarrestar las fuerzas tensiles.

La disfunción de la sínfisis púbica ocurre cuando la articulación se torna suficientemente relajada y permite que se desarrolle una inestabilidad pélvica. Snelling fue el primero en describir esta condición, en 1870, y a pesar de las múltiples revisiones del tema que se han hecho no existe unanimidad sobre los criterios diagnósticos y tampoco para evaluar alternativas terapéuticas. Es frecuente encontrarse esta patología en algunos grupos poblacionales como las mujeres gestantes. En especial, durante el segundo y tercer trimestres, cuando se presentan en aproximadamente el $89 \%$ de los casos. Para sospechar el diagnóstico es frecuente encontrar dolor a la palpación de la sínfisis púbica y de las articulaciones sacroiliacas. Puede haber limitación para los arcos de movimiento de la cadera, en especial durante la rotación externa y abducción. El tratamiento de esta patología requiere un abordaje multimodal con analgésicos, terapia física y, en algunos casos, rehabilitación continua.

\section{Articulaciones facetarias}

La articulación cigoapofisiaria (facetaria) hace parte de unidad funcional vertebro-ligamentaria junto al disco vertebral y la banda tendinosa posterior. Se ha encontrado que tiene un papel fundamental en el curso natural de la enfermedad discal degenerativa y en el desarrollo del canal lumbar estrecho (teoría de KirkaldyWillis). Se denomina enfermedad facetaria al proceso inflamatorio multifactorial sobre esta articulación sinovial inicialmente descrito, en 1911, por Goldthwaith (5).

Es frecuente encontrar la disfunción (inflamación) de la articulación facetaria en muchos pacientes con espondilopatía degenerativa, en especial en aquellos que cursan con alteraciones en el balance sagital, debido a cambios en la biomecánica donde el centro de gravedad ya no se encuentra sobre el cuerpo vertebral, sino que se desplaza hacia los elementos más posteriores. Estos segmentos no se encuentran diseñados filogenéticamente para soportar esta carga mecánica, lo que lleva a una pérdida de la función y a un cambio estructural (hipertrofia ósea, formación quistes facetarios y desarrollo de estenosis foraminal).

En ocasiones, durante el transcurso de la historia esta patología ha pasado inadvertida, debido a su desconocimiento y al amplio espectro clínico que puede tener (dolor referido en la región glútea y miembros inferiores). Sin embargo, se había sugerido que un dolor de características mecánicas que aumenta con la extensión y la rotación puede ser sugestivo de esta patología. De ahí que el uso de algunas imágenes diagnósticas, como la gammagrafía ósea y los 
bloqueos percutáneos permitan un diagnóstico de esta enfermedad; pero son insuficientes como herramienta de tamizaje rápida en el momento de la valoración clínica. Por ello, en 2004, Acevedo (5) describió una nueva maniobra, constituida por la fase I, con el paciente acostado en posición de decúbito supino con los miembros inferiores en posición de reposo; la fase II, en la cual se realiza una flexión forzada de la articulación de la cadera, con la pierna extendida y tomando como apoyo la parte más inferior de la pierna, y la fase III, en que se le pide al paciente que realice fuerza hacia abajo y con la mano apoyada en la parte inferior de la pierna. El examinador opone una fuerza opuesta a la anterior precipitando dolor axial en el segmento lumbar de paciente con disfunción facetaria. Esta prueba es una excelente herramienta clínica no invasiva asequible y de tamizaje de enfermedad facetaria (sensibilidad del 95\% y especificidad del 96\%) que se puede buscar en pacientes antes de ser llevados a artrodesis lumbar para evaluar su impacto en el transcurso del tiempo.

\section{Valoración de la discapacidad}

En la patología degenerativa espinal no se pueden representar los desenlaces satisfactorios únicamente en términos imagenológicos donde se mida el grado de fusión vertebral o de corrección del balance espino-pélvico. Dado que una de las repercusiones más importantes para el paciente con espondilosis y dolor lumbar crónico es la limitación en su calidad de vida y el control sintomático, una variable de interés para representar los desenlaces de los pacientes llevados a intervención quirúrgica debe incluir la escala visual análoga para el dolor y el grado de discapacidad que genera su patología. Dentro de las escalas más utilizadas en neurocirugía se encuentra la de Oswestry, descrita hace ya más de 30 años en la literatura. Fairbank y Pynsent (16) revisaron la literatura con una búsqueda sistemática sobre las diferentes versiones de esta escala y evidenciaron que todavía constituye una herramienta rigurosa y válida para aproximar el grado de limitación funcional de los pacientes.

\section{Conclusiones}

La artrodesis lumbar es un procedimiento que cada día tiene una mayor prevalencia en nuestro medio. Es importante tener claras las indicaciones para aplicar este tipo de intervenciones, lo que permite una mejor selección de los pacientes. La enfermedad facetaria, sacroilitis y la disfunción de la sínfisis púbica pueden ser entidades que si no se tratan de forma oportuna pueden perpetuar el dolor de forma postoperatoria. Por lo tanto, se deben buscar de forma activa desde la valoración prequirúrgica para poder establecer qué pacientes tienen mayor probabilidad de cursar con dolor o disfunción persistente

Así mismo, es importante realizar una adecuada valoración biomecánica empleando las diferentes mediciones (incidencia pélvica, lordosis lumbar, pendiente sacra e inclinación pélvica) que constituyen el estudio del balance espino-pélvico para así poder establecer el impacto en los desenlaces funcionales y de dolor.

Estos elementos mencionados constituyen factores pronósticos que impactan de forma favorable los desenlaces funcionales en la literatura internacional de los pacientes con enfermedad degenerativa de la columna lumbar.

\section{Referencias}

1. Last A, Hulbert K. Chronic low back pain: Evaluation and management: Am Fam Physician [internet]. 2009; 79(12):1067-74. Disponible en: https://www.aafp.org/af $\mathrm{p} / 2009 / 0615 / \mathrm{p} 1067 . \mathrm{html}$

2. Von Korff M, Crane P, Lane $\mathrm{M}$, et al. Chronic spinal pain and physical-mental comorbidity in the United States: results from the national comorbidity survey replication. Pain. 2005; 113:331-9.

3. Salvetti M de G, Pimenta CA, Braga PE, Corrêa CF. Disability related to chronic low back pain: prevalence and 
associated factors. Rev Esc Enferm USP. 2012 Oct; 46 Spec No:16-23.

4. Adams MA. Biomechanics of back pain. Acupunct Med. 2004 Dec; 22(4):178-88.

5. Acevedo JC. Síndrome facetario lumbar: Nuevo signo de diagnóstico clínico. Rehabilitación (Madr). 2004; 38(4):168-74.

6. Liliang $\mathrm{PC}$, Lu $\mathrm{K}$, Liang $\mathrm{CL}$, Tsai YD, Wang KW, Chen HJ. Sacroiliac join pain after lumbar and lumbosacral fusion: findings using dual sacroiliac join blocks. Pain Med. 2011; 12(4):565-70. doi: https://doi.org/10.1 111/j.1526-4637.2011.01087.x

7. Ames CP, Smith JS, Scheer JK, Bess S, Bederman SS, Deviren V, et al. Impact of spinopelvic alignment on decision making in deformity surgery in adults: A review. J Neurosurg Spine. 2012 Jun; 16(6):547-64.

8. Acevedo JC, Pérez JC. Correlación entre los resultados clínicos de los pacientes con dolor lumbar crónico, tratados con artrodesis lumbosacra y las variables biomecánicas de la unidad funcional lumbosacrococcígea. Bogotá: Unidad de Neurocirugía, Hospital Universitario San Ignacio; 2013.

9. Lamartina C, Berjano P, Petruzzi M, et al. Criteria to restore the sagittal balance in deformity and degenerative spondylolisthesis. Eur Spine J. 2012; 21(Suppl 1):27-31. doi: https://doi.org/ 10.1007/s00586-012-2236-9

10. Kjaer P, Bendix T, Sorensen JS, Korsholm L, Leboeuf-Yde C. Are MRI-defined fat infiltrations in the multifidus muscles associated with low back pain? BMC Med. 2007 Jan 25; 5:2. 11. Hildebrandt J. [Musculature as a source of back pain]. Schmerz. 2003 Dec;17(6):412-8.

12. Weinstein JN, Lurie JD, Tosteson TD, Hanscom B, Tosteson AN, Blood
EA, et al. Surgical versus nonsurgical treatment for lumbar degenerative spondylolisthesis. N Engl J Med. 2007; 356(22):2257-70.

13. Teichtahl AJ, Urquhart DM, Wang Y, Wluka AE, Wijethilake P, O'Sullivan R, Cicuttini FM. Fat infiltration of paraspinal muscles is associated with low back pain, disability, and structural abnormalities in communitybased adults. Spine J. 2015 Jul 1; 15(7):1593-601.

14. Acevedo JC, Quintero ST. Scale of diagnostics (SI5) for the sacroiliac joint dysfunction: Pilot study. Rev Soc Esp Dolor. 2014; 21(3):123-30. doi: http://dx.doi.org/10.4321/S1134-8 0462014000300002

15. Guan F, Sun Y, Zhu L, Guan G, Chen M, Chi Z, et al. Risk factors of postoperative sacroiliac joint pain for posterior lumbar surgery: $\geq 2$-year follow-up retrospective study. World Neurosurg. 2018 Feb; 110:e546-e551.

16. Fairbank JC, Pynsent PB. The Oswestry Disability Index. Spine. 2000; 25(22):2940-52. 